\title{
Beyond Accreditation: Issues in Healthcare Quality
}

Feroz Ikbal

\begin{abstract}
In the last few years, there is an increased interest among Indian Healthcare institutions to get accredited from bodies, such as national accreditation board for hospitals and healthcare providers (NABH), Joint Commission International (JCl), Australian Council on Healthcare Standards (ACHS), and college of american pathologists Laboratory accreditation programme (CAP), etc. Hospital administrators, clinicians, academicians, promoters of the hospitals, policy makers and even government feel that accreditation is a panacea for all the problems associated with healthcare quality. But with the incidence of fire in one of the $\mathrm{NABH}$ accredited hospital in a metropolitan city, questions began to be asked on the correlation between quality and accreditation. Most of the hospitals use accreditation as a promotional tool, rather than a tool for continuous quality improvement. Often the entire focus of quality in a hospital is confined to the process of accreditation and re-accreditation. Time has come to think on the entire process of accreditation of hospitals in India, though it has a history of less than a decade. This paper intends to discuss various issues of quality in hospitals, outside the realms of accreditation. Need for strengthening and re-engineering the accreditation is also discussed. Accreditation essentially identifies the capability of the hospital to deliver quality care. It does not assure that hospitals delivers quality care. This aspect of accreditation has been often forgotten by the various stakeholders in healthcare. In this paper, an attempt is made to discuss other issues of quality, such as spurious drugs, quality of biomaterials, such as stents and biomedical equipments, quality of human resources, etc. which are often neglected by health institutions in its obsession to accreditation.
\end{abstract}

Keywords: Quality, Healthcare institution, Obsession, Accreditation.

How to cite this article: Ikbal F. Beyond Accreditation: Issues in Healthcare Quality. Int J Res Foundation Hosp Healthc Adm 2015;3(1):1-4.

\section{Source of support: Nil}

Conflict of interest: None

\section{INTRODUCTION}

Hospital services in India are highly unregulated and fragmented. Eighty percent of healthcare services are

\section{Assistant Professor}

Centre for Hospital Management, School of Health Systems Studies, Tata Institute of Social Sciences, Mumbai, Maharashtra India

Corresponding Author: Feroz lkbal, Assistant Professor Centre for Hospital Management, School of Health Systems Studies, Tata Institute of Social Sciences, Mumbai, Maharashtra India, Phone: 8655324590, e-mail: feroz.ikbal@tiss.edu ferozikbal@yahoo.co.in provided by private sector, which ranges from small clinic of a quack to multispeciality hospitals, which attracts medical value travelers. The facilities in government sector vary from a sub-center to All India Institute of Medical Sciences (AIIMS).

There are serious discussions about the quality of care provided in hospitals, both in private and public sector. There are reports of medical negligence, medical error, poor patient safety, etc. in Indian hospitals. Institute of medicine (1998) in its report reveals that between 44,000 and 98,000 people die in United States due to medical errors every year. ${ }^{1}$ Number of deaths due to medical errors in India is anybody's guess as we have poor reporting systems and disclosure norms. Many a times if the disease cannot kill a person, treatment does.

Licensing and regulation by the government, certification by approved bodies and voluntary accreditation have been used as tools to improve and standardize the healthcare across the world. Among the three accreditation is generally considered to be the best tool to improve the patient safety and quality.

Hospital accreditation has been defined as 'A selfassessment and external peer-assessment process used by healthcare organizations to accurately assess their level of performance in relation to established standards and to implement ways to continuously improve'. ${ }^{2}$ Since, late 1990's, various stakeholders in Indian health sector have been advocating for accreditation of Indian healthcare facilities. National Accreditation Board for Hospitals and Healthcare Providers (NABH), a constituent board of Quality Council of India (QCI), was set-up to establish and operate accreditation program for healthcare organizations in India was established in the year 2006. Before $\mathrm{NABH}$ was established, Indraprastha Apollo Hospitals obtained the prestigious accreditation from Joint Commission International (JCI) in the year 2005. Since 2006, over 150 hospitals have obtained NABH accreditation and several hundred's are in the line. Around 13 hospitals have obtained JCI accreditation.

The important question which needs to be asked is whether getting accreditation the finishing point of race toward quality. Activity of quality management department in any hospital these days are focused only on the agenda of accreditation. But there are several issues of healthcare quality which are currently beyond accreditation which this paper intends to discuss. 


\section{REVIEW OF LITERATURE}

O'Connor PT and Wolff AM (1993) feels Hospital accreditation has been criticized for its emphasis on structure and documentation. Less attention is given to the clinical process and outcomes of care. To make accreditation process become a meaningful part of day-to-day hospital management, four approaches are considered. These includes the development of industrial type quality assurance programs that detect negative patient outcomes and improve patient care, The appointment of a Quality Assurance/Accreditation Coordinator with appropriate authority, the establishment of an effective Quality Assurance/Accreditation committee, and the continuous review of accreditation standards through normal committee function and department review and trial surveys. Such strategies will enable hospital accreditation to develop beyond a paper exercise and to provide the foundation for excellence in healthcare delivery. ${ }^{3}$

Greenfield, and Braithwaite in their study try to identify and analyze research into accreditation and accreditation processes. A multi-method, systematic review of the accreditation literature was conducted and 66 studies were retrieved and analyzed. The results, examining the impact or effectiveness of accreditation were classified into 10 categories: professions' attitudes to accreditation, promote change, organizational impact, financial impact, quality measures, program assessment, consumer views or patient satisfaction, public disclosure, professional development and surveyor issues. They concluded that attempts are being made to develop evidence for the effectiveness of accreditation. ${ }^{4}$

Hinchcliff et al examined 122 empirical studies that examined either the processes or impacts of accreditation programs in healthcare. Study components were recorded, including: dates of publication, research settings, levels of study evidence and quality using established rating frameworks, and key results. A content analysis was conducted to determine the frequency of key themes and subthemes examined in the literature and identify knowledge-gaps requiring research attention. There was not much strong evidence about the effectiveness of health service accreditation. ${ }^{5}$

Pomey et al discuss hospital accreditation as a tool for organizational change in France. According to this study, preparations for accreditation provided an opportunity to reflect non-hierarchically on the treatment of patients and on the hospital's operational modalities by creating a locus for exchanges and collegial decision making. These preparations also led to giving greater consideration to results of exit surveys and to committing procedures to paper, and were a key opportunity for introducing a continuous quality program. However, this study expresses doubts on cost of returns on accreditation. ${ }^{6}$

Ravi Mariwalla expresses doubt whether accreditation will improve healthcare delivery or whether it will be a mere documentation process. His concern is, once the accreditation was conferred, the hospital can slip back into old ways of functioning and worse, the strain of complying made staff go into a 'relax mode' that made service levels reach a low. Administrators as well as the media, experts and observers became particularly critical of the JCI approach when the Institute of Medicine publications brought to the fore the 'lack of safety' and the 'likelihood of harm during treatment' in healthcare institutions. ${ }^{7}$

\section{STATEMENT OF THE PROBLEM}

Accreditation has become the panacea for the poor quality and safety issues in Indian hospitals. The incidence of fire and subsequent death of around 100 people including patients and employees at AMRI Hospital, an $\mathrm{NABH}$ accredited hospital have raised serious questions on correlation between quality and accreditation. This paper intends to discuss the certain issues pertaining to quality, but which are beyond the broader dimensions of accreditation. Also, there is a need to understand what is covered in accreditation and what it does not stand for.

\section{MATERIALS AND METHODS}

This study has used secondary data from various textual sources. Various documents from newspapers, journals, healthcare magazines, Accreditation documents have been reviewed. Data analysis is through textual analysis.

\section{DISCUSSION AND FINDINGS}

Structure, process and outcome are the components of quality in healthcare system as per Donabedian. Most of the accreditation systems like JCI, NABH and ACHS have deliberated elaborately on these three components. The structure includes physical infrastructure, manpower, materials including medicines and biomaterials, and machinery including biomedical equipments. The processes in the hospital include diagnostic, therapeutic, administrative and supportive service delivery. But there are a few issues which affect the quality of healthcare delivery, which is vaguely covered in the accreditation which requires more serious attention. These include as follows:

\section{Spurious Drugs}

Hospitals spend roughly 25 to $40 \%$ of their annual operating budget on medicines. Safe medical has been widely 
discussed in NABH accreditation. Five R's of right medication include right patient, right medication, right dose, right time and right route. But unfortunately hospitals to a larger extend cannot have control over the quality of medicine which they are using. According to central drug standard control organization, an official body under ministry of health and family welfare only $0.3 \%$ of drugs in the Indian market is only spurious. ${ }^{8}$ But, several unofficial studies put the percentage of spurious drugs to as high as $20 \%$. Any drug, from an antibiotic to a pain medication, can be spurious. In 2007, fake drug makers sold more than 600 different types of branded, generic and over-the-counter drugs and used improved packaging to make their fake and spurious goods harder to detect. The spurious drugs act came into effect from August 2009 with the objective of eliminating the menace of manufacturing and sales of spurious drugs in the country. But, unfortunately like several other legislations in the country, though it appeared to be very stringent, it did not brought down the menace of spurious drugs to a larger extend.

\section{Biomedical Devices}

In India, Biomedical devices industry is highly unregulated and quality of biomedical devices used is highly dubious, including the imported devices. Ramachandran (2004) discusses the proposal to set up an Indian Medical Devices Regulatory Authority. Almost all countries that have a medical device industry have policies and regulatory processes or mechanisms in place. While the assurance of the quality of any marketed product is the responsibility of the manufacturer, the state also has the responsibility to protect the right of the consumer. In the case of medical devices, with potential health risks, the responsibility is even greater. In this scenario, it is indeed amazing that there is virtually no regulatory system in the country that ensures the reliability of these devices given that, like a drug or a vaccine, medical devices too, particularly those that are implanted in the human system, have attendant health risks. The more sophisticated is the underlying technology, the more complex becomes its calibration and operation and more serious are the potential errors due to malfunctioning and consequent harmful effects on a patient's clinical status. Therefore, like any drug, a medical device also requires to be evaluated for its quality, efficacy, reliability and safety before and after procedure for approval for its use in public health. If the device is implantable, it would also require systematic and rigorous preclinical and clinical studies, much like a drug. ${ }^{9}$

India lacks any kind of regulatory framework for certification, quality assurance, safety evaluation and postmarket surveillance of both imported and indigenous medical devices. Even the Drug Controller General of India (DGCI) does not have any mandate to regulate the medical devices market and the use of the devices in medicine. Apparently, the practice followed by the DGCI is to refer to matters relating to medical devices to the Indian Council of Medical Research (ICMR) on a case-to-case basis. Some low-technology devices like thermometers and weighing machines seek certification from the Bureau of Indian Standards (BIS) — for ISI marking-and that too is optional. Some imported high-tech devices, approved or cleared by their country of origin, as done by the United States' Federal Drug Administration (FDA), are apparently permitted to be marketed in India.

Many of the Indian hospitals use refurbished medical equipments, which can also cause potential harm to the patients. Some experts have opined that India has become the dumping ground for medical devices, implants and equipments. The exorbitant price and nexus between the physician-supplier is also serious concern with respect to medical devices particularly with respect to Drug Eluding Stents (DES). After dimensions of quality includes affordability and accessibility.

\section{Poor Industrial Relations}

The year 2011 to 2012 saw the issue of poor industrial relations in hospitals in India. Thousands of nurses across India have started an indefinite strike for better pay and conditions even in hospitals which have JCI and NABH accreditation. Nurses form the single largest professional group in hospitals. They are directly involved in the patient care. An unsatisfied internal customer can never satisfy an external customer. Historically unorganized, Indian nurses have formed a new union, creating over 400 branches in 2 months. Enquiry commissions by some state governments have revealed that many of the hospitals are not even paying the statutory minimum wages to the nurses. Many of the nurses feel they are being tortured and are not provided reasonable accommodation and quality food. Similar grievance has been raised by paramedical staff and supportive staff of hospitals. Hospitals being highly labor intensive needs to look into the industrial relations more seriously than ever before along with getting accredited.

There are other issues of quality which needs to be looked into while hospitals go in for accreditation. Over emphasis on documentation can be one weakness of accreditation. The transformation from 'letters' to 'spirit' is of great importance in quality improvement through accreditation. Another issue is the role of consultants in the accreditation program. Many of the hospitals depend too much on consultants for the accreditation program. Undoubtedly consultants bring in technical expertise 
and can improve the process. But hospitals needs to understand their own inherent strength and weakness, and should develop their own team for the continuous improvement. Quality and credibility of assessors of NABH will also be discussed in the near future. Often most of the assessors are practicing managers in hospitals. So, many independent observers doubt there can be quid pro quo in the assessment and final accreditation. Also efforts should be made to make the assessing team with multidisciplinary backgrounds of medicine, nursing and management.

There are several tools which can be used as tools to improve the quality beyond various accreditations. These are as follows:

\section{Medical Audit}

Medical audit or clinical audit is the comparison of actual practice against agreed, documented, evidence based standards with the intention of improving patient care. ${ }^{10}$ Once corrective action has been taken about problems identified through a review process, performance is remeasured after an appropriate time period. Though most of the accreditation programs includes medical audit, it is not done with the right rigor in the Indian hospitals.

Donabedian model: The Donabedian model is a conceptual model that provides a framework for examining health services and evaluating quality of care. The model was developed by Avedis Donabedian in 1966, which comprises of structure, process and outcome. ${ }^{11}$ The structure refers to the physical infrastructure, man power, materials and equipments. The core process in hospital includes diagnostic processes and therapeutic processes. These processes will be supported by other processes, such as administration, supportive services, engineering and maintenance, etc. The most important expected outcome is cure of the patient, improvement in the condition, death, left against medical advice. Other outcomes include profit or surplus, market share, utilization and efficiency of services.

Bench marking: Benchmarking is the process of comparing one's business processes and performance metrics to industry bests or best practices from other companies. In modern day management, the concept was introduced by Xerox in the year 1986. Benchmarking can be used as a tool to improve the processes in hospitals. In fact, hospitals can also benchmark their services with other industries for improving their supportive services.

\section{CONCLUSION}

Accreditation undoubtedly is an important tool in quality improvement. Many of the new hospital projects as well expansion plans are working for accreditation at the planning and designing stage itself. Few of the government hospitals are also already accredited and many of the state governments are taking efforts to get their hospitals accredited. But many existing hospitals both at the government and private sector cannot go for the accreditation as their physical infrastructure is not fit enough to go for the accreditation, processes not standardized and outcome not measured.

But accreditation is only component of quality in healthcare. Getting accredited and sleeping on that laurel till the time of re-accreditation can be dangerous for the safety of the patient and quality of the service as we have seen in some hospitals which are accredited. If we examine history of academic discipline of management at a given time there will some concept which will be widely discussed, but eventually fade out. Hope accreditation in hospitals do not have the fate of concepts like BPR, Bench Marking, Six sigma and other concepts.

\section{REFERENCES}

1. Institute of medicine, 2000; Bates et al, 1997.

2. Rawlins R. Hospital accreditation is important. BMJ 2001 Mar 17;322(7287):674

3. O'Connor PT, Wolff AM. Preparation for hospital accreditation: an efficient and practical approach. Australian Clinical Rev 1993;13(4):1.p.57-63.

4. Greenfield D, Braithwaite J. Health sector accreditation research: a systematic review. Int J Quality in Health Care 2008;20(3):172-183.

5. Hinchcliff R, Greenfield D, Moldovan M, Westbrook JI, Pawsey M, Mumford V, Braithwaite J (4 October). Narrative synthesis of health service accreditation literature. BMJ Quality and Safety Online First. doi:10.1136/bmjqs-2012-000852.

6. Pomey MP, Contandriopoulos AP, Francois P, Bertrand D. Accreditation: a tool for organizational change in hospitals. Int J Health Care Quality Assurance 2004;17(3):113-124.

7. Mariwalla R. Regulation in healthcare: improved care delivery or just documentation? Express Healthcare 2009 September.

8. Central drugs standard control organisation. Report on Countrywide Survey for Spurious Drugs' Directorate General of Health Services, Ministry of Health \& Family Welfare, Government of India 2009.

9. Ramachandran R. Monitoring medical devices'. Frontline magazine 2004 Mar 27-Apr 9;21(7).

10. Ferris M. 'Clinical audit tools and validation' Clinical Audit Conference, Westminster, London. 12-13th February 2002.

11. Donabedian A. Evaluating the quality of medical care. The Milbank fund quarterly 1966;44(3):166-223. 